\section{La salud de los hombres en la Región de las Américas ${ }^{1}$}

Se basa en "Perfil epidemiológico da saúde masculina na Regiaõ das Américas: uma contribução para o enfoque de gênero", documento elaborado por Ruy Laurenti, Cassia Maria Buchalla, M. Helena P. de Mello Jorge, Maria Lúcia Lebraõ y Sabina L. D. Gotlieb, de la Facultad de Salud Pública de la Universidad de São Paulo, Brasil, en 1998.
El análisis de la mortalidad, la morbilidad y otros indicadores tradicionales de salud de hombres y mujeres en la mayor parte de los países de las Américas indica una "desventaja masculina" que no se relaciona con el momento en que los datos fueron obtenidos. Hay claras diferencias entre los sexos en el sentido de que los hombres tienen menor esperanza de vida y mayor mortalidad en prácticamente todas las edades y por casi todas las causas. Sin embargo, los indicadores de morbilidad — medidos por la demanda de servicios y por los estudios de población sobre la mayor incidencia de episodios agudos de enfermedades, número de internaciones hospitalarias y duración de la convalecencia- indican una mayor frecuencia en las mujeres.

Si bien no hay duda de que algunas de las diferencias de mortalidad se deben a factores determinantes biológicos, hay ciertos comportamientos específicos de cada sexo regidos por factores culturales y sociales que perjudican su salud. Hoy día el término sexo (masculino o femenino) se refiere a la condición biológica natural gobernada por leyes genéticas que determinan una anatomía corporal y una fisiología específica, mientras que el término género (hombre o mujer) se refiere a una construcción social que ha asignado papeles definidos a cada sexo a lo largo de la evolución histórica de la humanidad. De ello se desprende que ciertos factores del comportamiento favorecen una mayor frecuencia de algunos trastornos, así como su mayor letalidad, en un sexo que en otro.

La duración de la vida individual (longevidad) como característica específica de la especie humana no ha cambiado a lo largo del tiempo, pero el promedio de vida de la población está aumentando debido a la disminución de la mortalidad en edades más jóvenes, tanto en los hombres como en las mujeres. Sin embargo, la proporción de longevos en la población masculina es menor que entre las mujeres en prácticamente todos los países de las Américas. En los países más desarrollados desde el punto de vista social, económico y de salud pública, como el Canadá y los Estados Unidos, hay un mayor porcentaje de ancianos que en los países en desarrollo y una diferencia más acentuada entre los sexos. Aunque no se cuenta con series históricas prolongadas de datos de la mayoría de los países de la Región, la dispersa y escasa información disponible permite verificar que la esperanza de vida de los hombres exhibe el mismo comportamiento que el promedio de vida. 


\section{ANÁLISIS DE LOS DATOS DE MORTALIDAD}

La comparación de la mortalidad de los hombres y la de las mujeres en diferentes edades desde el punto de vista biológico, en contraposición al social o conductual, no es sencilla si se realiza aisladamente. En cada edad la mortalidad obedece a un conjunto de causas sujetas a diferentes factores, ya sean biológicos o no. En lo que se refiere a algunas dolencias, como la arteriosclerosis y ciertos tipos de tumores malignos, no sería inapropiado afirmar que la edad es un factor biológico de riesgo.

Por lo común, la mortalidad de los varones menores de 1 año es mayor que la de las niñas en todos los países de las Américas y semejante a la observada en otras zonas del mundo. Esta diferencia puede evaluarse mejor si se analizan las causas de muerte. Como las causas naturales son por lo general semejantes en ambos sexos, las razones de esa diferencia se encuentran principalmente en las causas externas, es decir, en el diferente grado de exposición de niños y niñas a accidentes y violencia.

En el documento de R. Laurenti et al., los autores analizaron los datos de mortalidad de hombres y mujeres correspondientes al período de 1981 a 1984 en países seleccionados de diversas subregiones de la Región de las Américas. La selección se basó en la calidad de las estadísticas vitales disponibles, consideradas completas si aportaban datos que incluían $90 \%$ o más de los acontecimientos anuales, e incompletas si los datos incluían menos de $90 \%$ de esos acontecimientos. En América Latina, se escogieron Colombia y Venezuela de la subregión andina; la Argentina, Chile y el Uruguay de la subregión del Cono Sur; Brasil y México, cada uno como subregión en sí mismo; Costa Rica, Guatemala y Panamá de Centroamérica; Cuba y Puerto Rico del Caribe de habla española; Barbados y Jamaica del Caribe de habla inglesa, y el Canadá y los Estados Unidos de América en América del Norte. Asimismo, se procuró que los datos tuvieran las siguientes características: disponibilidad en publicaciones oficiales internacionales, porcentajes bajos de causas mal definidas frente al total de defunciones y número suficiente de defunciones para evitar fluctuaciones estadísticas.

El análisis de la mortalidad según sexo y edad en las Américas alrededor de 1985 mostró un patrón común a algunos países. Entre los adultos jóvenes de 15 a 24 años de edad, la mortalidad llegó a ser hasta $200 \%$ más alta que la de las jóvenes, probablemente porque la concentración de muertes en ese grupo de edad se suele relacionar con las causas externas. En una serie temporal de mortalidad según la edad, se observó su disminución constante en ambos sexos aunque la disminución fue menor en los hombres que en las mujeres.
Si bien el exceso de mortalidad masculina en todos los grupos de edad no es una característica exclusiva de la Región de las Américas, llama la atención que en los países menos desarrollados la mortalidad de los grupos de edad más jóvenes fuera mayor en ambos sexos y que la masculina también fuera mayor que la femenina. A edades más avanzadas, particularmente por encima de los 60 años, las tasas de mortalidad y las diferencias entre los sexos fueron muy semejantes en todos los países.

\section{CAUSAS DE MUERTE}

De manera general, en el perfil de la mortalidad masculina en las Américas se destacaron como causas más importantes las enfermedades cardiovasculares - sobre todo, la enfermedad isquémica del corazón- y los tumores malignos -en especial, de estómago, pulmón y próstata- Las causas externas agregaron una característica importante a dicho perfil pues aparecieron siempre entre las cinco causas principales de muerte, especialmente los accidentes de vehículo de motor y los homicidios. Los factores de riesgo de estas causas se asocian a ciertos comportamientos o al nivel socioeconómico y estarían, por lo tanto, más ligados a los aspectos de género que a los biológicos. La hipótesis resulta útil cuando se buscan los factores socioculturales que puedan explicar las causas del perfil de la mortalidad masculina aunque en los niños, especialmente los menores de 1 año, todo indica que los factores biológicos son predominantes, si no los únicos.

\section{Tumores malignos}

La mortalidad masculina por tumores malignos siempre excedió a la femenina excepto por los tumores de cuello uterino y de mama. Los tumores malignos fueron más frecuentes a medida que aumentaba la edad y hubo una inversión de la diferencia de mortalidad entre los sexos con el paso de los años. Así, en los países estudiados la mortalidad femenina fue constantemente más alta en el grupo de 15 a 44 años, pero hubo mayor mortalidad de los hombres de 45 a 64 años. Otro aspecto interesante fue que la razón entre las tasas de mortalidad del grupo de 65 o más años y las del de 15 a 44 años tuvo un incremento mucho mayor entre los hombres. También resultó notable que las tasas de mortalidad masculina por varios tipos de tumores malignos fueron más altas en los países de las Américas con peores condiciones socioeconómicas. Es posible que ello se haya debido a la menor accesibilidad a los servicios de salud preventivos o curativos. 
Entre los tumores malignos, el cáncer de estómago fue la causa más importante de defunción $(17,5 \%$ del total) y la mortalidad fue mayor en los hombres en todas las ciudades estudiadas. Hay quienes aducen que los factores de riesgo que más influyen en la frecuencia de este cáncer en los hombres son el mayor valor promedio de tejido gástrico vulnerable y la mayor cantidad de alimentos de impacto carcinógeno que ingieren, independientemente de las condiciones ambientales.

Los tumores malignos de pulmón como causa de muerte en los hombres se ubicaron en primero o segundo lugar en todos los países de las Américas y la diferencia entre los sexos fue bastante mayor que la del cáncer gástrico. Por otra parte, la frecuencia del cáncer de próstata aumentó gradualmente cuando se midió mediante las tasas de mortalidad. En general, la mortalidad por este tipo de tumor siguió en importancia a la de los tumores de pulmón y estómago. Llama la atención el incremento de las tasas a partir de los 55 años de edad, las cuales fueron 8 a 10 veces más altas, con un crecimiento rápido y acentuado en los grupos de 65 a 74 años y de 75 o más años. Ya que es difícil determinar con seguridad los factores de riesgo y establecer programas enfocados hacia la prevención, resulta fundamental el diagnóstico precoz de la enfermedad por medio del análisis del antígeno prostático específico en la sangre.

\section{Enfermedades cardiovasculares}

La mortalidad masculina por enfermedades cardiovasculares fue también mayor que en las mujeres. El exceso de defunciones de hombres por enfermedad isquémica del corazón y otras cardiopatías se destacó en todos los países, especialmente en el grupo de 45 a 64 años de edad. También interesa señalar que las muertes por esta enfermedad ocurrieron en edades más tempranas y se desencadenaron más rápidamente en los hombres. La marcada incidencia y alta mortalidad por enfermedad isquémica del corazón y su alta mortalidad pueden subrayarse como características epidemiológicas principales de la salud de los hombres no solo en los países de las Américas sino en el resto del mundo, con excepción de algunos países muy pobres del continente africano. Asimismo, esta enfermedad constituye la principal causa básica de muerte masculina en la mayor parte de los países de la Región.

Aunque en los países seleccionados no predominó claramente la mortalidad masculina por enfermedades cerebrovasculares, todos los estudios muestran que el sexo masculino y los antecedentes familiares son factores críticos de riesgo. Como esos factores no pueden alterarse, debe considerarse la gran importancia de modificar el comportamiento con el fin de adoptar un modo de vida más sano. Además, es necesario controlar la hipertensión arterial - que tiene mayor prevalencia en los hombres y aumenta con la edad-y reducir el alcoholismo mediante un enfoque de género.

\section{Causas externas}

En contraste con las causas naturales, el análisis de la distribución de las tasas de mortalidad por causas externas según el sexo permite apreciar que las diferencias tienen poco o nada que ver con el factor biológico. En los hombres la tendencia parece estar asociada a factores del comportamiento que los llevan a exponerse al peligro de accidentes o violencia y morir. Esa diferencia entre los sexos fue comparable en todos los países de la Región, con tasas siempre más elevadas en los hombres. Además, resulta notable que las causas externas afectaron principalmente a los hombres, lo que hace que los accidentes y la violencia den cuenta del mayor número de años potenciales de vida perdidos (APVP). Cabe observar que la frecuencia de las defunciones por esa causa se está desplazando hacia grupos de edad más jóvenes. Las tasas de mortalidad por suicidio fueron más altas en los hombres en todos los países; en cuanto al homicidio, cuyo origen generalmente se vincula con las desigualdades sociales, fue una causa básica de defunción entre adolescentes y adultos jóvenes del sexo masculino. En la actualidad, los factores ambientales y culturales parecen ser los que explican mejor las diferencias apuntadas, pues son los que afectan más a los comportamientos relacionados con una mayor exposición al riesgo de morir por causas externas en ambos sexos.

Por otra parte, en los certificados de defunción de los diferentes países de las Américas no suele especificarse cuáles son las muertes por accidentes de trabajo. Aunque puede ser difícil clasificar con exactitud los diferentes tipos de causas externas, está claro que el sexo masculino seguirá teniendo la mayor representatividad en el total de muertes por accidentes y violencia, independientemente de su distribución.

\section{Sida}

El sida es actualmente la enfermedad que mejor permite considerar la importancia de las cuestiones de género en su diseminación. Esta epidemia comenzó hace menos de 20 años, pero ha experimentado cambios epidemiológicos importantes y la historia natural de la enfermedad se modifica a medida que se conoce. En realidad se trata de un conjunto de pequeñas epidemias que van cam- 
biando según los hábitos culturales y de comportamiento prevalecientes.

Las características locales de la epidemia dependen básicamente de los modos de transmisión predominantes en cada zona. En general, en los grandes centros urbanos sobresalieron la transmisión homosexual y por uso de drogas inyectables, con mayor incidencia en adultos jóvenes, especialmente de la población masculina. En las ciudades portuarias, además de esos dos modos de transmisión, se registraron casos de transmisión heterosexual y una alta prevalencia de VIH entre las trabajadoras del sexo. Al iniciarse el decenio de 1990, la transmisión heterosexual había adquirido importancia creciente en las Américas, pero la mayor incidencia seguía correspondiendo a los hombres.

$\mathrm{Al}$ principio de la epidemia la mayor parte de los casos ocurrían en hombres homosexuales o bisexuales. Luego la epidemia se extendió entre los usuarios de drogas inyectables -incluso mujeresy pasó a tener mayor relieve la transmisión heterosexual. La transmisión homosexual es importante en gran parte de los países que notifican casos de sida y, por el hecho de que la epidemia se inició entre los hombres, el número de enfermos con VIH es todavía mayor entre la población masculina, especialmente en el grupo de 20 a 55 años.

La intensa mortalidad en hombres jóvenes es uno de los indicadores de la fuerza de la epidemia. En contraste con las principales causas de muerte que inciden en las edades extremas, el sida afecta principalmente a los adultos jóvenes, y aunque la enfermedad ha llegado a afectar a otros grupos, como las mujeres y los niños, su importancia relativa no ha disminuido en el sexo masculino. La enfermedad se subnotifica por desconocimiento de que es obligatorio hacerlo pero además, en todos los países y en distinto grado, se omite o se demora voluntariamente la notificación de casos de sida como causa de muerte.

A pesar de que el sida tiene su mayor incidencia en el sexo masculino, no sería admisible afirmar que es una infección para la que los hombres están más predispuestos biológicamente que las mujeres. Tampoco puede considerarse que la progresión de la enfermedad esté ligada al sexo. Una característica que aumenta la probabilidad de transmisión entre los hombres es que, en general, los varones tienen actividades sexuales más frecuentemente que las mujeres. Igualmente, algunos comportamientos específicos promovidos por influencias sociales o culturales alteran la vulnerabilidad individual; por ejemplo, la sensación de ser invencibles que suelen tener los hombres adolescentes y adultos jóvenes hace que se expongan al riesgo de contraer infecciones.

Entre la población heterosexual, la vulnerabilidad al sida aumenta con las prácticas sexuales riesgosas, entre ellas, tener un gran número de compañeros sexuales y relaciones sexuales sin protección. En cuanto a los usuarios de drogas, si bien en la infección por VIH no hay una propensión basada en el género, debe señalarse que la drogadicción es más frecuente en la población masculina que en la femenina.

\section{MORBILIDAD}

En gran parte de los países de las Américas, los datos de morbilidad son escasos, sobre todo en los países menos industrializados. Aun así, los registros de morbilidad también mostraron diferencias entre los sexos. Las mujeres asistieron más asiduamente a los servicios ambulatorios para consultar por sus síntomas de malestar quizá por tener una percepción más aguda de las modificaciones de su cuerpo. También mostraron una mayor predisposición a buscar la ayuda necesaria y, frecuentemente, fueron responsables de cuidar a los hijos y llevarlos a los servicios de salud. Sin embargo, esa tendencia de las mujeres a acudir a consulta no se observó cuando se analizaron los datos de algunas enfermedades de notificación obligatoria en las que la incidencia está ligada a la ocupación. En cuanto a datos sobre hospitalización, no hubo grandes diferencias entre los sexos, exceptuados los partos y las complicaciones del embarazo, el parto y el puerperio.

En las causas no relacionadas con el sexo, hubo más internaciones de mujeres por tumores malignos, más internaciones de hombres por trastornos asociados al tabaquismo o al consumo de alcohol y preponderancia del sexo masculino en las correspondientes localizaciones anatómicas de los tumores. Las lesiones y envenenamientos originaron trastornos muy desfavorables para el sexo masculino y produjeron un cuadro semejante al de la mortalidad por causas externas.

Los datos obtenidos resaltaron la importancia de las consecuencias en la salud del consumo excesivo de alcohol, especialmente en los hombres. Solo la violencia ocasionó diferencias de mortalidad o morbilidad entre los sexos más elevadas que el alcoholismo. En los países de América Latina, donde los hábitos y comportamientos poco saludables relacionados con el tabaquismo y el consumo de alcohol están fuertemente asociados con el "machismo", resulta de gran importancia analizar el tema con un enfoque de género.

\section{MODOS DE VIDA}

Si bien las enfermedades vinculadas con ciertos modos de vida fueron comunes a ambos sexos, 
se manifestaron con más intensidad en el masculino por factores culturales. Los ejemplos clásicos son el hábito de fumar y el cáncer de pulmón en los hombres. En las Américas, los factores de riesgo de las enfermedades no infecciosas que afectan a la salud de los hombres adultos se relacionan con los modos de vida - especialmente los tumores malignos de estómago, pulmón y próstata, los problemas cardiovasculares por complicaciones de la arteriosclerosis y la hipertensión, y las consecuencias de la violencia por homicidio o de los accidentes de vehículos de motor- y se sienten con más intensidad en la población de menores ingresos y grado de escolaridad. En consecuencia, la incidencia y la mortalidad por esas causas continúan siendo altas en la población general que no tiene acceso a la información sobre los riesgos que afectan a su salud.

El análisis de los datos muestra que la desventaja masculina a lo largo del tiempo es el resultado de la acción de diferentes factores de riesgo ligados a los aspectos biológicos y a los de género, es decir, culturales, sociales y de comportamiento. Una investigación específicamente formulada permitiría describir y analizar algunos aspectos del comportamiento de los hombres y determinar si interfieren con la salud cuando se asocian a factores biológicos o si son independientes de los mismos. Aunque hasta el momento todos los estudios muestran que el sexo masculino y los antecedentes familiares son factores importantes de riesgo sobre los que no se puede actuar, debe considerarse la gran importancia del factor conductual que sí puede modificarse y permite así adoptar un modo de vida sano. En consecuencia, deberían tenerse en cuenta los factores culturales al planificar las acciones educativas de los programas de salud destinados a prevenir las enfermedades relacionadas con los modos de vida de las distintas zonas de la Región.

\section{SYNOPSIS}

\section{Men's health in the Americas}

In mortality, morbidity, and other traditional indicators of health of men and women, there is a "male disadvantage" in most countries of the Americas, according to a 1998 report by Ruy Laurenti and other researchers from the School of Public Health of the University of São Paulo, Brazil. Their analysis found that men have shorter life spans and higher mortality rates at nearly every age and for almost every cause, due to risk factors related to "sex" (the men's biological status) and also to their "gender" (their roles and behaviors as determined by society).

The report's authors compared mortality and morbidity data for men and women for the period of 1981 to 1984 in selected countries of various subregions of the Americas. The researchers found higher mortality for men in all age groups. Among the most common causes of death for men were cardiovascular illnesses, malignant tumors, and AIDS. The category of external causes, especially motor vehicle accidents and homicides, was always among the five leading causes of death among males. The only exception were boys under 1 year of age, for whom biological factors were the most important. The risk factors for those external causes are more closely tied to "gender" behaviors than to "sex."

For causes of morbidity not determined by the person's sex, women had more hospitalizations because of malignant tumors. Men had more hospitalizations linked to smoking and to drinking alcohol and had more of the related tumors. Men also suffered more often than women from injuries and poisonings. Illnesses connected to lifestyle were more common among men, especially males with lower incomes and less education.

Laurenti and the other researchers conclude that in the various regions of the Americas, "machismo" and other gender factors ought to be considered in planning the educational component of health programs that seek to prevent lifestyle-related illnesses. In addition, the authors suggest that research designed to describe and analyze some behaviors of men could determine if those actions themselves are harmful to health or if they are damaging when they are associated with biological factors. 